\title{
An Experimental Study of Chinese Shopping Related Sharing Behaviors
}

\author{
Min $\mathrm{Li}^{1}$, Jun Zhang ${ }^{1}$, Zhengjie Liu ${ }^{1}$, and Graham I. Johnson ${ }^{2}$ \\ ${ }^{1}$ Sino-European Usability Center, Dalian Maritime University, \\ Dalian, 116026 P.R. China \\ ${ }^{2}$ Consumer Experience Research, NCR Corporation \\ Dundee, UK \\ minleely@gmail.com, \{William,liuzhj\}@dlmu.edu.cn, \\ graham.johnsonencr.com
}

\begin{abstract}
Social media has become an increasingly important venue for social interaction and communication. Most previous research have shown the information sharing on social media platforms, but few focus on shopping related sharing (SRS) information and how these information influence consumer decision-making. Through a combination of interviews with observations in our lab to the users of social media and online shopping sites, we want to find answers to the question: what are the factors that influence Chinese consumers' SRS behaviors? It was discovered that the factors like why, what, whom, where, when etc. have significant influence to Chinese consumers' SRS behaviors. The study offers insights into the relationship between SRS and Chinese consumer decision-making, and design implications are discussed.
\end{abstract}

Keywords: Social media, shopping, sharing, social influence, consumer decision-making.

\section{Introduction}

Social media is now flourishing worldwide and plays a more and more important role in most people's lives as a means of information acquirement and sharing. It is notable that more and more shopping related contents appeared along with the combination of social media and E-commerce, for example, pictures with links from other online shopping websites and consumer generated contents about the whole shopping experience. Businesses also create public profiles or make advertisements on social media platforms to broadcast their products and attract consumers.

Consumer decision-making process is complicated and influenced by many factors, and now more and more affected by social media information. However, what is the relationship between social sharing behaviors and consumer decision-making? $\mathrm{We}$ propose a conceptual framework to indicate how social influence impact consumer decision-making process, and want to recognize the role social media plays on Chinese consumer decision-making from some point of view by studying SRS behaviors. By doing this, we can get the first hand knowledge of SRS and help us further study how SRS influence consumer decision-making. 
The remainder of this paper is structured as follows. First we introduce the background and related work. Then we propose the conceptual framework to guide our study, and then describe research methods and processes. Next we describe the findings, followed by a short discussion of the design implications of our work for business and those in the interaction design community. Finally, we highlight the conclusions and next steps of our research work.

\section{Background and Related Work}

Recent years, the studies on commerce-oriented reviewing and sharing platforms (CRSP) primarily addressed the problems of summarizing and analyzing online reviews. For example, researchers conducted data mining on reviewing text generated by consumers rather than measuring the rating scores[1].Some researchers studied the problem of generating a "rated aspect summary" of short comments, which is a decomposed view of the overall ratings for the major aspects so that a user could gain different perspectives towards the target entity [2]. Some other studies addressed the problem of sentiment classification of the comments [3][4]. Although researchers have explored many aspects of social media platforms (e.g. blogs, Facebook, and Twitter etc.), most of which have primarily focused on the social network connections, user activities (like what, how, why, where and when) and privacy concerns [2] [5]. Some researchers paid more attention to the information contents. They classified the contents into some categories, analyzed the political opinions by data mining tools and conducted sentiment analysis of the comments [6].

In literature [7], the researchers showed that E-commerce had been largely affected by social media. Researchers have proposed that consumers share shopping information on social media platforms through status messages [8]. They presented types of questions asked by users, and found that five percent of the questions asked were shopping related. However, they didn't explore in-depth how shopping related information could influence purchasing decisions, which was our research focus. Jansen et al. examined Twitter usage for brand sentiment, and gave a report about information sharing among $20 \%$ of status updates by Twitter participants, and specifically indicated that people shared generally positive opinions about brands [9]. Park et al. focused on sharing of daily deal URL on Twitter, and they adopted quantitative analysis method to analyze sharing behaviors of daily deal information [10]. They provided evidence of the potential contribution of Twitter in improving the daily deal sales performance. There are also researchers with focuses on advertisements and business public profiles on social media [11]. However, they still didn't pay much attention on Chinese social media, so we are interested, from an HCI and user experience perspective, in the different ways in which social media is being used for commerce in order that subsequent designs and affordances of such services can be improved.

Previous studies paid less attention to the relationship between SRS and consumer decision-making process. We propose a conceptual framework based on the model [12] proposed by Thompson et al. to illustrate social influence on three sub-phases of consumer decision-making process (need recognition, information collection and after 
purchase evaluation). Basing on the conceptual framework, we try to investigate social sharing behaviors of Chinese consumers and the motivations underlying the behaviors. In the future work, we will explore how the sharing behaviors on different social media platforms influence decision-making.

\section{Conceptual Framework}

Many scholars have argued that a virtual community is capable of disseminating product knowledge and awareness and influencing community members' purchase behavior [10][13]. On the basis of previous research [12], in which Thompson et al. proposed the consumer decision process model to illustrated the core decision-making process. The process begins with need recognition, then information search, alternative evaluation, purchase and after purchase evaluation. We focus on information sharing related parts and propose a conceptual framework to illustrate factors influence the three steps of decision-making process, see details in Figure 1. Factors related to need recognition (NR), information collection intention (ICI) and after purchase evaluation (ACI) include perceived benefit (PB) and perceived risk (PR). Factors related to PR include trust (T), privacy concerns (PC) and social influence (SI). And SI is a factor influence both T and PC.

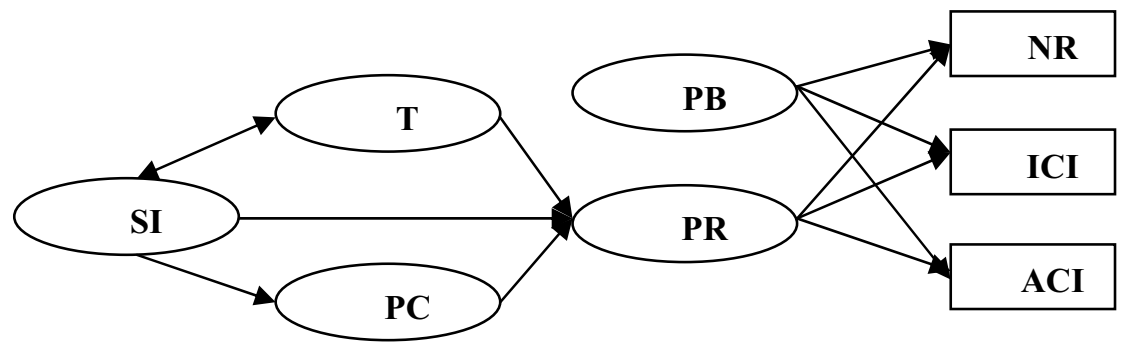

Fig. 1. Conceptual framework

PB refers to perceived benefit that is expected to result from the three steps. PR refers to perceived risk that is defined as the probability of any loss that can occur. $\mathrm{T}$ refers to trust that is defined as the belief that the result will be good. PC refers to privacy concerns that are defined as personal data or information privacy, users care about the unclear results from information leakage. SI refers to social influence that users may impacted by social media and/or other members in the social networks. We emphasize SI factor which is one of the most important factors in consumer decisionmaking process. And in this paper, we try to understand SRS behaviors and the social influence behind the behaviors.

\section{$4 \quad$ Methodology and Process}

We adopted lab-based sample user study. We recruited active users of social media sites and online shopping sites for interview and observation in our lab. They were 
asked to talk about their experience in SRS behavior and simultaneously show us on a PC how they were doing it. For example, we asked participants to answer whether or not share shopping related information and how, when, where and who to share. They talked about the latest SRS behaviors on social media platforms or other offline conditions. By using this method, we gained the knowledge about real online buyers' shopping and sharing experience quickly and directly.

We recruited 30 participants via an agency and got 29 qualified from them which were numbered from 1 to 29 respectively, see details in Table 1 . We collected data of participants' SRS behaviors, demographic information, applied social media applications and online shopping behaviors by screenshot, audio recording and note taking. Each session lasted around 1.5 hours.

Table 1. Distributions of basic information of the participants

\begin{tabular}{lll}
\hline Category & Item & Value \\
\hline Gender & Male/Female & $14 / 15$ \\
Age group & $18-24 / 25-30 / 31-36$ & $9 / 10 / 10$ \\
Education degree & Junior college student/ Undergraduate/ & $15 / 12 / 2$ \\
& Postgraduate & \\
Online shopping websites & Taobao.com & 29 \\
used & Groupbuy websites & 11 \\
& Dangdang.com & 5 \\
& Paipai.com & 4 \\
& Amazon.cn & 3 \\
& 360buy.com & 2 \\
& Others & 4 \\
\hline
\end{tabular}

We used grounded theory to analyze data and had some interesting findings during the study and summarized the factors that influence SRS behaviors, like why and what to share, where to share, whom to share with, and when to share. We present the findings of the study in details below.

\section{$5 \quad$ Results}

We will present the findings related to Chinese SRS behaviors both online and offline. We focus on the following aspects: 1) why and what to share, 2) where to share, 3) whom to share with, and 4) when to share.

Why and What to Share. Participants usually do not want to share shopping related information, because they have privacy and personal image concerns $(20 / 29$, means 20 participants mentioned this point out of total 29 participants). But the situation is not unalterable, they will share when there are rewards for doing so, e.g., sharing groupbuy related information.

Participants are afraid that it may harm their privacy by sharing on social platforms. They do not want friends on their online social network to know what they bought, neither personal products (gifts for lovers, health medicine, etc.) nor daily goods. They regard shopping as a private activity: 
"I don't want to share shopping information with others because it is personal thing." Participant 19, female.

Dholakia et al. proposed several social influence variables that could affect users' behaviors, such as social identity [8]. In our study, we found Chinese consumers cared a great deal about their personal images both online and offline, which was consistent with social identity. On one hand, some kind of information can improve image, leaving a good impression to others that the person is skillful, special, etc. On the other hand, some kind of information can damage image, like fake information, so participants do not want to take the risks introduced by sharing.

It is notable that personal image and privacy concerns may be weakened when there are rewards for doing so, e.g., sharing group-buy related information. We saw that the group-buy information was shared on Renren by participants 11 . If any friend clicked the hyperlink to go to the group-buy website and at last bought the product, the participant could get a discount from the group-buy website in the form of a voucher.

Where to Share. Participants tend to share particular kinds of information on different platforms with different market positioning or segment. There are four major kinds of sharing platforms being mentioned frequently in our study: 1) built-in consumer reviewing platform and attached commerce-oriented social network services (SNS) platforms on online shopping websites, 2) relationship-emphasized or socially-oriented SNS platforms, 3) instant messenger (IM) platforms, and 4) dedicated consumer reviewing platforms.

Taobao is a typical online shopping website with a built-in consumer reviewing platform. Buyers can search the comments left by other buyers on it before they make purchase decisions. After the deal, they can also make a comment related to the products they bought. An example of the attached commerce-oriented SNS platforms is i.taobao.com, which is attached to Taobao and encourages users to participate in social networking activities as they do on other SNS platforms. Participants do have SRS behaviors on these platforms, but we find that the motivations of the SRS behaviors are rewards in the forms of reward points and discounts provided by Taobao but not social purposes to recommend better products to other users on the platform.

Renren, Sina Weibo and Tencent Weibo are typical examples of socially-oriented social media platforms. Even active users seldom share shopping related contents on these platforms. The main reason is concern on personal image and privacy. Another reason is that they regard shopping related contents so trivial that too much sharing may cause information overload to others.

Almost all the few SRS behaviors (5/29) we observed on these platforms were group-buy related, because strong-tie friends are regarded as the potential group-buy partners. This will potentially bring benefits to both the sharer and the friends:

"I will share the group-buy food to my friends on Renren, because I can get the reward of 5 Yuan from the website if my friend also buys this food through my link. That's a win-win. Why not?" Participant 8, female.

QQ, Fetion, MSN Messenger and Wangwang are typical examples of IM platforms. QQ is the most popular IM tool in China. Almost every Chinese web user has 
one account at least. Fetion (developed by China Mobile) and MSN Messenger also have large number of users in China. Wangwang is an IM tool attached to Taobao, and is most widely used between buyers and sellers when they discuss the deal related information. In our study, we found that most of the SRS behaviors happened among QQ-group members and users even have dedicated shopping group names like "shopping" and "group-buy". Participants regard this kind of platforms as appropriate places for SRS probably because QQ IM communication is so popular and timely that there is a great chance that there will be always a few friends already online and can get feedback immediately.

Dianping (www.dianping.com) and Mogujie (www.mogujie.com) are typical dedicated consumer reviewing social platforms in China, like Epinions in west countries. Users use these platforms to exchange shopping related information and comments. The majority of the information shared on these platforms is systematic and in-depth comments related to the products, even hyperlinks to online shopping websites, and users tend to seek for helpful information from these platforms in their shopping decision-making processes.

Whom to Share with. We will summarize people whom participants are more likely to share with. We find that there are mainly three kinds, 1) friends nearby in the physical world, 2) people that can potentially bring benefits, and 3) people that have similar preferences or experiences with the participant.

We find that participants are more likely to share with friends geographically nearby in the physical world (28/29). Apart from using IM tools like QQ, in more cases participants share by face to face or phones, because they live near to each other or have the chance to meet frequently in daily lives and know each other well:

"The products will be delivered to my company on working days, and my colleagues can see what I have bought, and discuss about them" Participant 14, female.

Participants are likely to share with those who can potentially bring benefits (8/29), e.g., share with potential group-buy partners. Participants told us that good products or services should be shared or recommended to others to bring benefits to them. But we think they also had concerns about the rewards for doing so based on our observations.

Participants are likely to share with those who have similar preferences or experiences with them $(5 / 29)$, for information from those people tends to be more valuable to them. The phenomena can be explained by social influence theory that users want to keep consistence with peers [14], which can verify the conceptual framework we proposed.

When to Share. We find that there are two main situations when participants share, one is when the quality and attached services of the products are very good or very bad, and the other is when participants just want to show themselves, especially when they buy special things.

Participants are more likely to share when the quality and attached services of the products are very good or very bad (16/29). If the product is very good and may be suitable for others, then the participant will suggest potential consumers to buy it. 
Sometimes participants share comments on bad products or services to others to express their negative feelings and also to remind other consumers not to buy it, and at this time the comments will be longer.

Sometimes participants just want to show themselves, especially when they have bought some special products. Participant 3 showed us a picture of a bought white mouse on his Qzone. He explained that the white mouse is bought online as the food for his pet python. He thought that the sharing was so unique and made him different from others.

\section{Discussion and Design Implications}

We have achieved a basic understanding of Chinese SRS behaviors based on the analysis of the qualitative data collected in the lab-based sample user study. We identified the factors that could influence SRS behaviors. It is notable that Chinese consumers are very much concerned about personal image online, but when there are economical rewards, the concerns may be weakened. We believe that market planners can make use of the insight to encourage consumers to share SRS information by giving them more rewards, such as coupons or discounts.

Chinese IM tools also should be mentioned, especially QQ. Because of its convenience for using and so large number of users, shopping related topics are diffusing frequently among QQ users and QQ group members. So, it is convenient to use IM tool to gather consumer needs to keep current consumers and attract potential consumers at the same time.

Some participants mentioned that often they can't find the right channel to access shopping related information except search engines like Google and Baidu. There are chances for companies to make better use of social media platforms to broadcast contents about their brands or products to more potential consumers. On another hand, recommendation system should give the most relevant and valuable SRS contents to the right people who really need them.

Another important aspect is peer's pressure. Consumers care much about the comments from nearby friends and people with similar preferences or experiences. Consumers want to keep consistent with peers, so they regard peers' reviews as most valuable. So, some mechanism should be considered to support interaction about SRS between geographically nearby strong-tie relationships.

\section{Conclusions and Next Steps}

We have achieved a basic understanding of SRS based on the analysis of the qualitative data collected in the lab-based sample user study. Four kinds of factors that influence SRS behaviors were identified. In the near future, we will discuss the relationship between SRS behaviors and consumer decision-making, and verify the conceptual framework we proposed. And next we will start quantitative and qualitative analysis of questionnaire data we collected online to enrich and verify the findings we've mentioned. And then we will also begin to address other social media 
platform, such as location-based services (LBS) applications, to study SRS behaviors and enrich our knowledge about the relationship between SRS and consumer decision-making.

\section{References}

1. Zhang, Z., Li, X., Chen, Y.B.: Deciphering Word-of-Mouth in Social Media: Text-Based Metrics of Consumer Reviews. ACM Transactions on Management Information Systems 3(1) (2012)

2. Nylander, S., Rudstrom, A.: Questions, inspiration, feedback, and contributions: How entrepreneurs network online. In: Proceedings of C\&T 2011, pp. 128-137. ACM Press (2011)

3. Mei, Q., Ling, X., Wondra, M., Su, H., Zhai, C.: Topic sentiment mixture: modeling facets and opinions in weblogs. In: Proceedings of WWW 2007, pp. 171-180. ACM Press (2007)

4. Popescu, A.M., Etzioni, O.: Extracting product features and opinions from reviews. In: Proceedings of the Conference on Human Language Technology and Empirical Methods in Natural Language Processing, pp. 339-346 (2005)

5. Gilbert, E., Karahalios, K.: Predicting tie strength with social media. In: Proceedings of CHI 2009, pp. 211-220. ACM Press (2009)

6. Song, J.X., Finin, T., Tseng, B.: Why we Twitter: understanding microblogging usage and communities. In: 9th WebKDD and 1st SNA-KDD 2007 Workshop on Web Mining and Social Network Analysis (webKDD/SNA-KDD 2007), San Jose, California, USA, pp. 56-65 (2007)

7. Turban, E., Bolloju, N., Liang, T.P.: Social Commerce: An E-Commerce Perspective. In: Proceedings of ICEC 2010, pp. 33-42. ACM Press (2010)

8. Morris, M.R., Teevan, J., Panovich, K.: What Do People Ask Their Social Networks, and Why? A Survey Study of Status Message Q\&A Behavior. In: Proceedings of CHI 2010, pp. 1739-1748. ACM Press (2010)

9. Jansen, B.J., Zhang, M., Sobel, K., Chowdhury, A.: Twitter power: Tweets as electronic word of mouth. Journal of the American Society for Information Science and Technology 60 (2009)

10. Park, J.Y., Chung, C.W.: When Daily Deal Services Meet Twitter: Understanding Twitter as a Daily Deal Marketing Platform. In: Proceedings of WebSci 2012, pp. 233-242. ACM Press (2012)

11. Cvijikj, I.P., Michahelles, F.: Understanding Social Media Marketing: A Case Study on Topics, Categories and Sentiment on a Facebook Brand Page. In: Proceedings of MindTrek 2011, pp. 175-182. ACM Press (2011)

12. Thompson, S.H., Yeong, Y.D.: Assessing the consumer decision process in the digital marketplace. The International Journal of Management Science 31, 349-363 (2003)

13. Dholakia, U.M., Bagozzi, R.P., Pearo, L.K.: A social influence model of consumer participation in network- and small-group-based virtual communities. International Journal of Research in Marketing 21, 241-263 (2004)

14. Moscovici, S.: Social influence and conformity. In: The Handbook of Social Psychology, vol. 2, pp. 347-412. Random House, NY (1985) 